\title{
Isotopic Inventory and Activity Calculations of Fukushima Daiichi Unit-1 Accident
}

\author{
Rowayda Fayez Mahmoud ${ }^{1}$, Mohamed Moustafa Abdu ${ }^{2}$ and Mohamed Kamal Shaat ${ }^{{ }^{*}}$ \\ ${ }^{(1)}$ Nuclear Reactors Department, Nuclear Research Center, Egyptian Atomic Energy Authority, Cairo, Egypt. \\ (2) Zewail City of Science\& Technology, Cairo, Egypt.
}

\begin{tabular}{|c|c|}
\hline ARTICLE INFO & ABSTRACT \\
\hline $\begin{array}{l}\text { Article history: } \\
\text { Received: } 27^{\text {th }} \text { Aug. } 2020 \\
\text { Accepted: } 1^{\text {st }} \text { Apr. } 2021\end{array}$ & $\begin{array}{l}\text { Nuclear characterization of the spent nuclear fuel in a reactor core is essential, especially } \\
\text { in case of severe accidents. The radionuclide inventory and its activity can assist in the } \\
\text { management of spent fuel handling, transport or reprocessing. }\end{array}$ \\
\hline \multirow{4}{*}{$\begin{array}{l}\text { Keywords: } \\
\text { Fukushima Daiichi accident, } \\
\text { Radionuclide inventory, } \\
\text { MCNPX 2.7, } \\
\text { Spent nuclear fuel } \\
\text { characterization, } \\
\text { Activity of spent nuclear fuel }\end{array}$} & $\begin{array}{l}\text { In this paper, the core of Fukushima Daiichi Unit-1(FD-U1) accident was modeled using } \\
\text { the Monte Carlo code (MCNPX 2.7) linked to the depletion calculation code CINDER'90 and }\end{array}$ \\
\hline & $\begin{array}{l}\text { ENDF/B-VII.0 cross section data library. The isotopic inventory and the activity of the } \\
\text { radionuclides for the burned fuel were calculated. The input to the code depends on the } \\
\text { previous evolution of the reactor core configurations, dimensions and material of the fuel } \\
\text { assemblies, initial uranium enrichment, fuel burn-up and reactor core operational history. }\end{array}$ \\
\hline & $\begin{array}{l}\text { The calculations were validated with experimental measurements which were carried out } \\
\text { by the Japan Nuclear Energy Safety Organization (JNES) and verified with published results } \\
\text { using ORIGEN2-code by Japan Atomic Energy Agency (JAEA). The validation and } \\
\text { verification results were in good agreement. }\end{array}$ \\
\hline & $\begin{array}{l}\text { The masses, activities, specific activities, half-lives and decay schemes for the actinides and } \\
\text { fission products were calculated at the time of the accident and after } 50 \text { years cooling time. } \\
\text { The calculations showed that, total activity of the burned fuel in the core at the time of the } \\
\text { accident was } 9.86 \mathrm{E}+19 \mathrm{~Bq} \text { and after } 50 \text { years was } 1.89 \mathrm{E}+17 \mathrm{~Bq} \text { and the higher inventory } \\
\text { concentration in the fuel was dominated by the trans-uranic elements. Also, the specific } \\
\text { activity in the core at the time of the accident and after } 50 \text { years cooling time was found to be } \\
1.84 \mathrm{E}+15 \mathrm{~Bq} / \mathrm{g} \text { and } 5.86 \mathrm{E}+12 \mathrm{~Bq} / \mathrm{g} \text {, respectively. These calculations are required for nuclear } \\
\text { characterization of the corium and in the estimation of the radiological consequences of the } \\
\text { source term in the environment. Also, the results can support the recovery program for } \\
\text { Fukushima Daiichi- Unit-1. }\end{array}$ \\
\hline
\end{tabular}

\section{1- INTRODUCTION}

On $11^{\text {th }}$ March 2011, Fukushima Daiichi Unit 1 (FD$\mathrm{U} 1$ ), was generating electricity when the earthquake occurred, it was shutdown automatically. The resulting tsunami halted the emergency core cooling, water levels in the reactor vessel started to drop from the top of the hot fuel, and steam began reacting with Zircaloy-4 cladding to produce large amounts of hydrogen and the core began to melt $[1,4,26]$.

Several published studies dealt with the calculation of core inventory and source term for nuclear reactor accidents using different methodologies and assumptions or through the measurements of the environmental samples around the reactor site. Knowing the inventory of the radionuclides of the burnt fuel is crucial to assess the estimation of the environmental consequences of radionuclide releases following a severe nuclear accident [11,13-15, 23-29].
The current U.S. high level waste inventory is dominated by the spent fuel produced from pressurized water reactors and boiling water reactors in commercial nuclear power plants. NRC has developed a beta version of a flexible performance assessment, Scoping of options and Analyzing Risk, Beta version ( $\beta$-SOAR) to provide risk and performance insights for a range of potential geological disposal options for high-level waste and spent nuclear fuel [10].

In this paper, the isotopic inventories and activities of the radionuclides of the burned fuel for FD-U1 accident was calculated at the time of the accident (scram time) and after 50 years cooling time using the Monte Carlo code MCNPX 2.7 and using the ENDF/B-VII.0 cross section data library [17-26]. The modeling depends on the history of the reactor core evolution until the last damaged core, and at the burn-up value reached before the reactor scram. The reactor fuel was modeled based on the previous operating period. The results were 
validated and compared with experimental measurements carried out by the Japan Nuclear Energy safety Organization (JNES) and verified with the calculations carried out by Japan Atomic Energy Authority (JAEA) using ORIGEN code [2,3].The information obtained are necessary for the characterization, management and decommissioning plan of the damaged core of FD-U1

\section{2- REACTOR CORE MODELING}

\subsection{Evolution of core configuration}

FD-U1 is a BWR MARK 3 type reactor operated by Tokyo Electric Power Company. The reactor core contained 400 fuel assemblies consisting of 332 (9x9-9) type B and 68(8x8-8) Type STEP2, the fuel is uranium dioxide and its total charge of uranium content is 69 ton. Each fuel assembly contains fuel pins in a square array and water channels that are all cotained in zircaloy-4 fuel channel box.

The control rods located outside the fuel assemblies are in cross-shaped arrangements of blades containing boron carbide $\left(\mathrm{B}_{4} \mathrm{C}\right)$. The fuel pins consist of low enrichment uranium oxide with an average enrichment of $3.7 \%$, enclosed and sealed in zirconium cladding tubes. The technical data of the FD-U1 is presented in Table (1) [5-19].

Table (1): Technical data of FD-U1

\begin{tabular}{lll}
\hline \multicolumn{3}{c}{ Core configuration } \\
\hline Total amount of uranium & 69 & ton \\
Reactor thermal Power & 1380 & $\mathrm{MW}_{\text {th }}$ \\
\hline \multicolumn{3}{c}{ Fuel assembly } \\
\hline Total number & 400 & \\
Channel box material & Zircaloy-4 & \\
Inner channel width & 13.05 & $\mathrm{~cm}$ \\
Outer channel width & 13.9 & $\mathrm{~cm}$ \\
\hline & Fuel rod & $\mathrm{cm}$ \\
\hline Diameter of pellet & 0.94 & $\mathrm{~cm}$ \\
Diameter of cladding & 1.1 & $\mathrm{~cm}$ \\
Thickness of cladding & $0.070(0.01)$ & \\
Number of fuel rods in one & $9 \times 9-9(\mathrm{water}$ & \\
fuel assembly & channel $)$ & $\mathrm{cm}$ \\
Gap radius & 0.48 & $\mathrm{~g} / \mathrm{cm}^{3}$ \\
Cladding material & Zircaloy-4 & $\mathrm{cm}$ \\
Fuel Rod Pitch & 1.45 & $\mathrm{~cm}$ \\
Fuel rod active length & 366 & $\mathrm{~cm}$ \\
Fuel density (97 \% of & 10.7 & \\
theoretical density) & $3.7 \%$ & \\
Enrichment & 6.56 & \\
Cladding density & 3.75 & \\
Water channel width & & \\
\hline
\end{tabular}

\subsection{Model description}

The core model of FD-U1 using MCNPX 2.7 code, was based on 400 assemblies of 9x9-9 type B and average enrichment of $3.7 \%$ with the preservation of the same mass amount of $\mathrm{UO}_{2}$. The number of fuel assemblies loaded in the last core configuration before the accident was 100 assemblies, which is about one quarter of the core. So, the last core consists of 100 fresh fuel assemblies and 300 burned assemblies with five different burn-ups. This core was operated at full thermal power of $1380 \mathrm{MW}_{\text {th }}$ for an operating period of 165 days and reached an average burn-up of about 25.82 GWd/MTU at 11 March 2011.

\subsubsection{Geometrical model}

MCNPX modeling of the fuel assembly and fuel rod geometry was based on the assumption of continuous bun at constant power for the fuel rod. Also, MCNPX calculations depend on using small time steps during irradiation compared to ORIGEN code which uses large time steps. The fuel assembly and fuel rod modeling are shown in Figure (1).
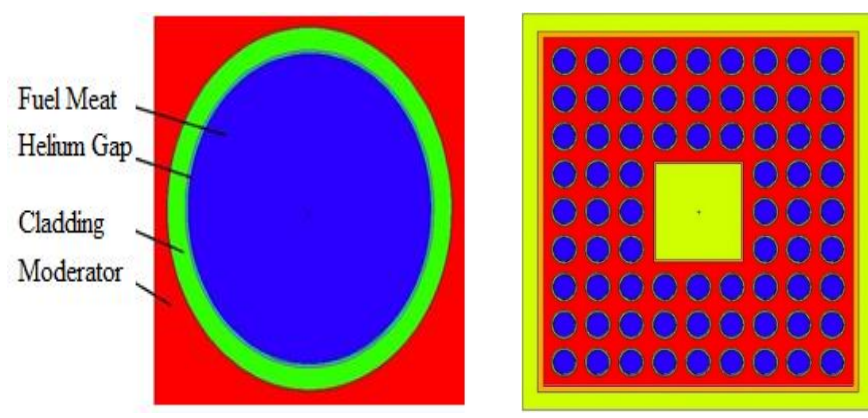

Fig. (1): MCNP modeling of fuel rod and fuel assembly 9x9-9 Type B

\subsubsection{Material composition of the core}

The initial atomic densities before the accident for cladding and channel box, Moderator and fuel meat composition are given in Table (2).

Table (2): Initial atomic densities for:

a) Zr-4 cladding and channel box

\begin{tabular}{lc}
\hline Element & Atom density (atoms/b.cm) \\
\hline $\mathbf{C r}$ & $7.5891 \mathrm{E}-5$ \\
$\mathbf{F e}$ & $1.4838 \mathrm{E}-4$ \\
$\mathbf{Z r}$ & $4.2982 \mathrm{E}-2$ \\
\hline
\end{tabular}

b) Moderator

\begin{tabular}{ccc}
\hline Void\% & Nuclide & Atom density (atoms/b.cm) \\
\hline \multirow{2}{*}{40} & H-1 & $3.0588 \mathrm{E}-2$ \\
& O-16 & $1.5294 \mathrm{E}-2$ \\
\hline
\end{tabular}


c) Fuel meat (wt. $\%=3.7)$

\begin{tabular}{lc}
\hline \multicolumn{1}{c}{ Element } & Atom density (atoms/b.cm) \\
\hline U-235 & $8.82 \mathrm{E}-4$ \\
U-238 & $2.27 \mathrm{E}-2$ \\
O-16 & $4.71 \mathrm{E}-2$ \\
\hline
\end{tabular}

\subsection{Model Validation}

The modeled core was validated and simulated for one fuel assembly and the results were compared with experimental measurements which were carried out by Japan Nuclear Energy Safety Organization (JNES) [2,3].

This experiment was carried out for samples of BWR 9x9-9 fuel assembly. The Isotopic Dilution Mass Spectrometry was used for sample measurements. The measured sample results for $\mathrm{U}$ and $\mathrm{Nd}$ isotopes were compared with our model calculations as given in Tables (3 and 4). From these Tables, it is clear that the obtained results using the MCNPX code are in close resemblance with the measured values by JNES.

Table (3): Comparison for isotopic inventories of Uranium

\begin{tabular}{rccc}
\hline Isotope ID & $\begin{array}{c}\text { MCNPX } \\
\text { (atoms/b.cm) }\end{array}$ & $\begin{array}{c}\text { MCNPX } \\
\text { (atoms/total U } \\
\text { atoms) }\end{array}$ & $\begin{array}{c}\text { Measured } \\
\text { (atoms/total U atoms), } \\
\text { JNES [11] }\end{array}$ \\
\hline U-233 & $8.70 \mathrm{E}-11$ & $3.88 \mathrm{E}-09$ & - \\
$\mathrm{U}-234$ & $5.94 \mathrm{E}-06$ & $2.65 \mathrm{E}-04$ & $3.00 \mathrm{E}-04$ \\
$\mathrm{U}-235$ & $2.39 \mathrm{E}-04$ & $1.07 \mathrm{E}-02$ & $1.03 \mathrm{E}-02$ \\
$\mathrm{U}-236$ & $1.54 \mathrm{E}-04$ & $6.85 \mathrm{E}-03$ & $7.00 \mathrm{E}-03$ \\
$\mathrm{U}-237$ & $1.30 \mathrm{E}-07$ & $5.81 \mathrm{E}-06$ & - \\
$\mathrm{U}-238$ & $2.20 \mathrm{E}-02$ & $9.82 \mathrm{E}-01$ & $9.82 \mathrm{E}-01$ \\
$\mathrm{U}-239$ & $5.98 \mathrm{E}-09$ & $2.67 \mathrm{E}-07$ & - \\
Total density & $2.24 \mathrm{E}-02$ & & \\
\hline
\end{tabular}

Table (4): Comparison for isotopic inventories of Neodymium

\begin{tabular}{cccc}
\hline Isotope ID & $\begin{array}{c}\text { MCNPX } \\
\text { (atoms/b.cm) }\end{array}$ & $\begin{array}{c}\text { MCNPX } \\
\text { (atoms/total U } \\
\text { atoms) }\end{array}$ & $\begin{array}{c}\text { Measured } \\
\text { (atoms/total U atoms), } \\
\text { JNES [11] }\end{array}$ \\
\hline Nd-142 & $1.01 \mathrm{E}-06$ & $4.51 \mathrm{E}-05$ & $5.67 \mathrm{E}-05$ \\
Nd-143 & $3.25 \mathrm{E}-05$ & $1.45 \mathrm{E}-03$ & $1.85 \mathrm{E}-03$ \\
Nd-144 & $5.62 \mathrm{E}-05$ & $2.51 \mathrm{E}-03$ & $3.51 \mathrm{E}-03$ \\
Nd-145 & $2.98 \mathrm{E}-05$ & $1.33 \mathrm{E}-03$ & $1.69 \mathrm{E}-03$ \\
Nd-146 & $3.16 \mathrm{E}-05$ & $1.41 \mathrm{E}-03$ & $1.73 \mathrm{E}-03$ \\
Nd-147 & $7.98 \mathrm{E}-08$ & $3.56 \mathrm{E}-06$ & - \\
Nd-148 & $1.56 \mathrm{E}-05$ & $6.96 \mathrm{E}-04$ & $8.92 \mathrm{E}-04$ \\
Nd-150 & $6.13 \mathrm{E}-06$ & $2.74 \mathrm{E}-04$ & $4.04 \mathrm{E}-04$ \\
Total density & $1.73 \mathrm{E}-04$ & & \\
\hline
\end{tabular}

\section{3- RESULTS AND DISCUSSIONS}

The total activity of the radionuclides for the source term of FD-U1 at the time of the accident was calculated using present MCNPX code, and grouped according to the NUREG-1465 classification which was presented at Table (5).

Table (5): Radionuclides classification of source term for F-D (U1)

\begin{tabular}{ll}
\hline Radionuclides grouping & $\begin{array}{l}\text { Activity } \\
(\mathbf{B q})\end{array}$ \\
\hline Nobel Gases (Xe, Kr) & $3.96 \mathrm{E}+18$ \\
Halogens (I, Br) & $4.00 \mathrm{E}+18$ \\
Alkali Metals (Cs, Rb) & $4.78 \mathrm{E}+17$ \\
Tellurium group (Te, Sb, Se) & $2.03 \mathrm{E}+18$ \\
Barium, strontium (Ba, Sr) & $3.85 \mathrm{E}+18$ \\
Noble Metals (Ru, Rh, Pd, Mo, Tc, Co) & $7.27 \mathrm{E}+18$ \\
Lanthanides ( $\mathrm{La}, \mathrm{Zr}, \mathrm{Nd}, \mathrm{Eu}, \mathrm{Nb}, \mathrm{Pm}, \mathrm{Pr}, \mathrm{Sm}, \mathrm{Y})$ & $1.34 \mathrm{E}+19$ \\
Cerium group (Ce, Pu, Np) & $3.45 \mathrm{E}+19$ \\
\hline
\end{tabular}

The initial amount of uranium fuel in the core (after the last reloading) before the accident was about 69 ton as shown in Table (1), and the calculated amount of the uranium fuel at the time of the accident (scram time) was about 66.51 ton as shown in Table (6). Hence, the rest of the uranium fuel mass was depleted for about 165 operating days. According to JAEA, the total estimated mass of the core composition was about 121.48 ton, which includes the depleted fuel, control rods and structural materials as shown at Table (6). The details of this estimation was calculated by using MCNPX code and presented in Tables (9 and 10) at the time of the accident and after 50 years from the accident.

Table (6): Estimated core structure materials inventory at the time of the accident

\begin{tabular}{lc}
\hline Material & Mass (ton) \\
\hline $\mathbf{U}^{\mathbf{a}}$ & 66.51 \\
$\mathbf{U O}_{2}{ }^{\mathbf{a}}$ & 75.77 \\
Fission Product $^{\mathbf{a}}$ & 2.49 \\
Zircaloy-4 (Clad) $^{\mathbf{a}}$ & 17.8 \\
Zircaloy (channel box) $^{\mathbf{a}}$ & 13.82 \\
Fe $^{\mathbf{b}}$ & 12.5 \\
$\mathbf{B}_{4} \mathbf{C}^{\mathbf{b}}$ & 0.59 \\
Inconel $^{\mathbf{b}}$ & 1 \\
Total $^{\text {a: MCNPX calculations }}$ & 121.48 \\
\hline b: Fumiya Tanabe [28] &
\end{tabular}


The activity of the calculated radionuclides at the time of the accident and after 50 years of fuel cooling using MCNPX 2.7 code were verified with JAEA calculations using the ORIGEN2 code $[2,3,19]$ as shown in Figures (2 and 3). The results were found to be in good agreement. It can also, be noticed that the activity and the specific activity of the major actinides after 50 years from the accident were reduced to $3.42 \mathrm{E}+16 \mathrm{~Bq}$ and to $2.04 \mathrm{E}+11 \mathrm{~Bq} / \mathrm{g}$, respectively. Moreover, the total activity and specific activity of the core melt are $1.89 \mathrm{E}+17 \mathrm{~Bq}$, and $1.56 \mathrm{E}+9 \mathrm{~Bq} / \mathrm{g}$, respectively, assuming a homogenized mixture of core melt.
Tables (7 and 8) present the inventories and corresponding activities of actinides and fission products for the core at the time of the accident and after 50 years calculated using MCNPX Code. We can notice from Tables (7 and 8) the important contribution of the precursors to the long-term inventory and high activity of ${ }^{237} \mathrm{~Np}\left(\mathrm{~T}_{1 / 2}=2.16 \times 10^{6}\right.$ years $)$. It can be assumed that ${ }^{237} \mathrm{~Np}$ precursors decay instantly, to account for the decay of ${ }^{241} \mathrm{Pu}\left(\mathrm{T}_{1 / 2}=14.4\right.$ years $)$ to ${ }^{241} \mathrm{Am}\left(\mathrm{T}_{1 / 2}=432.2\right.$ years) and then decay to ${ }^{237} \mathrm{~Np}$.So, their initial amounts can be added to the initial amount of ${ }^{237} \mathrm{~Np}$. Although ${ }^{245} \mathrm{Cm}\left(\mathrm{T}_{1 / 2}=8500\right.$ years $)$ is also a precursor to ${ }^{237} \mathrm{~Np}$, it only occurs in small amount in spent fuel and its contribution is negligible.

\section{- JAEA- ORIGEN2 EAEA-MCNPX}

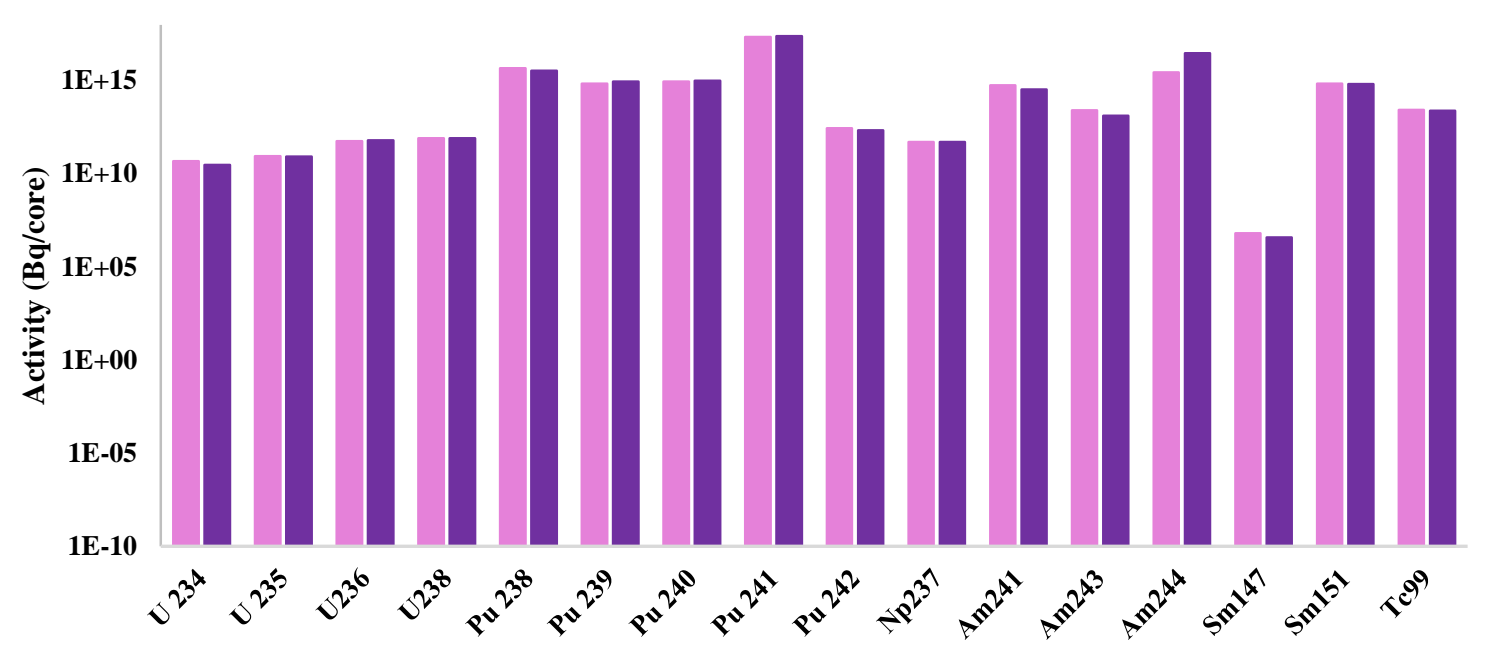

Fig. (2): Activity of the radionuclides at reactor scram

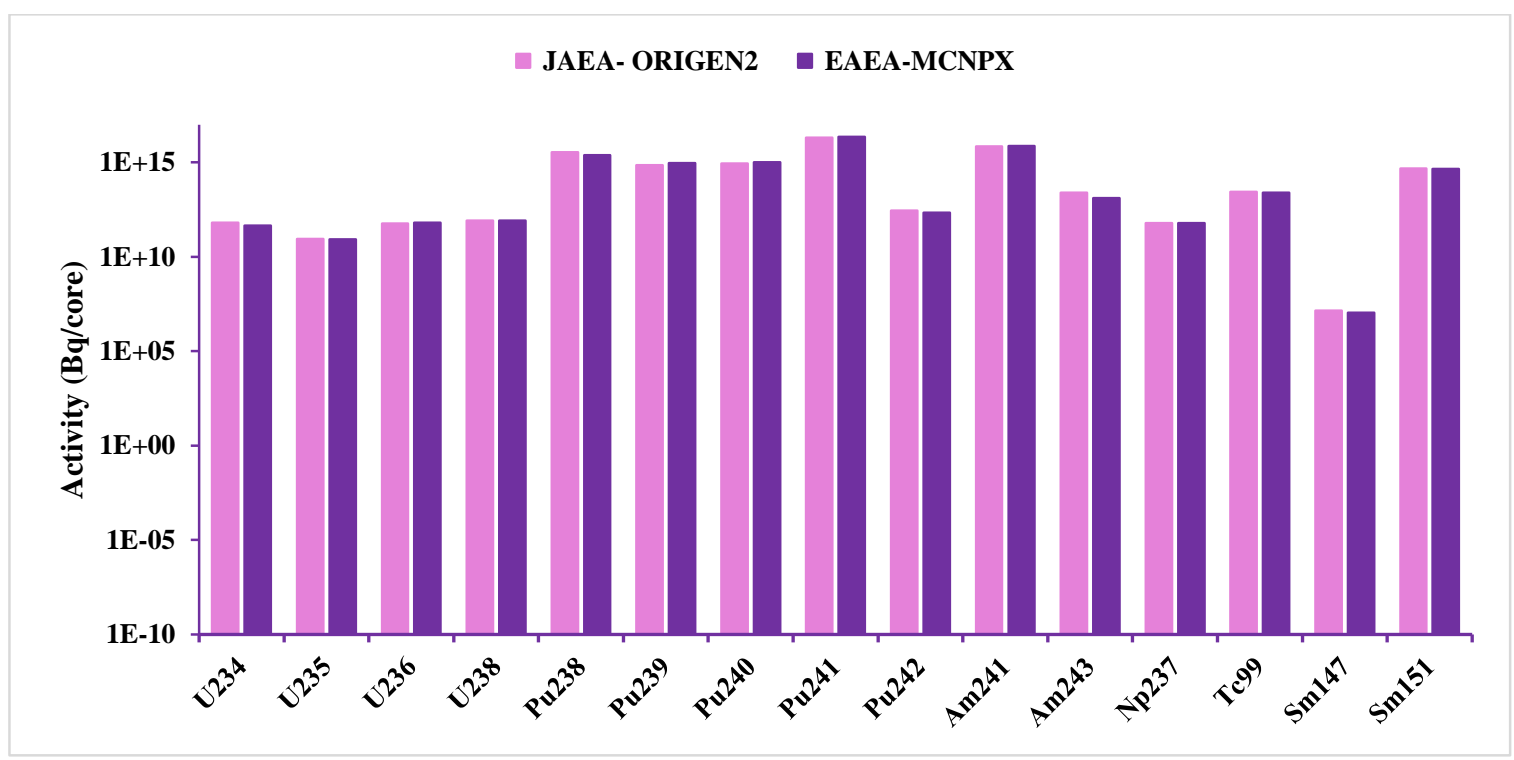

Fig. (3): Activity of selected radionuclides after 50 years from scram 
Table (7): Inventory and activity of the actinides and fission products for the core at the time of reactor scram

\begin{tabular}{|c|c|c|c|c|}
\hline Radionuclide & $\begin{array}{c}\text { Activity } \\
\text { (Bq/core) }\end{array}$ & $\begin{array}{c}\text { Half-life } \\
\text { (T1/2) }\end{array}$ & Decay Mode & $\begin{array}{c}\text { Inventory } \\
\text { (g/core) }\end{array}$ \\
\hline U-239 & $2.79 \mathrm{E}+19$ & $23.45 \mathrm{~min}^{*}$ & $\beta-(\mathrm{Np} 239)$ & $2.25 \mathrm{E}+01$ \\
\hline Np-239 & $2.78 \mathrm{E}+19$ & $2.356 \mathrm{~d}^{*}$ & $\beta-(\mathrm{Pu} 239)$ & $3.24 \mathrm{E}+03$ \\
\hline Xe-133 & $2.81 \mathrm{E}+18$ & $5.243 \mathrm{~d}$ & $\beta-(\mathrm{Cs} 133)$ & $4.06 \mathrm{E}+02$ \\
\hline I-135 & $2.62 \mathrm{E}+18$ & $6.57 \mathrm{~h}^{*}$ & $\beta-(\mathrm{Xe135})$ & $2.00 \mathrm{E}+01$ \\
\hline Te-132 & $1.94 \mathrm{E}+18$ & $3.204 \mathrm{~d}$ & $\beta-(\mathrm{I} 132)$ & $1.73 \mathrm{E}+02$ \\
\hline I-131 & $1.37 \mathrm{E}+18$ & $8.0252 \mathrm{~d}$ & $\beta-(\mathrm{Xe131})$ & $2.98 \mathrm{E}+02$ \\
\hline $\mathrm{Xe}-135$ & $1.12 \mathrm{E}+18$ & $9.14 \mathrm{~h}$ & $\beta-(\operatorname{Cs} 135)$ & $1.20 \mathrm{E}+01$ \\
\hline U-237 & $9.38 \mathrm{E}+17$ & $6.752 \mathrm{~h}$ & $\beta-(\mathrm{Np} 237)$ & $3.11 \mathrm{E}+02$ \\
\hline Sm-153 & $4.59 \mathrm{E}+17$ & $46.284 \mathrm{~h}$ & $\beta-(E u 153)$ & $2.80 \mathrm{E}+01$ \\
\hline $\mathrm{Np}-238$ & $3.20 \mathrm{E}+17$ & $2.099 \mathrm{~d}$ & $\beta-(\mathrm{Pu} 238)$ & $3.34 \mathrm{E}+01$ \\
\hline Pm-147 & $2.73 \mathrm{E}+17$ & $2.6234 \mathrm{y}^{*}$ & $\beta-(\operatorname{Sm} 147)$ & $7.96 \mathrm{E}+03$ \\
\hline $\mathrm{Pu}-241$ & $2.48 \mathrm{E}+17$ & $14.329 \mathrm{y}$ & $\beta-(A m 241)$ & $6.48 \mathrm{E}+04$ \\
\hline $\mathrm{Pu}-243$ & $2.14 \mathrm{E}+17$ & $4.956 \mathrm{~h}$ & $\beta-(A m 243)$ & $2.23 \mathrm{E}+00$ \\
\hline Cs-134 & $2.11 \mathrm{E}+17$ & $2.0652 \mathrm{y}$ & $\beta-(\mathrm{Ba} 134)$ & $4.41 \mathrm{E}+03$ \\
\hline Cs-137 & $1.98 \mathrm{E}+17$ & $30 \mathrm{y}$ & $\beta-(\mathrm{Ba} 137)$ & $6.14 \mathrm{E}+04$ \\
\hline Sr-90 & $1.56 \mathrm{E}+17$ & $28.79 \mathrm{y}$ & $\beta-(Y 90)$ & $2.99 \mathrm{E}+04$ \\
\hline Am-642 & $1.10 \mathrm{E}+17$ & $16.02 \mathrm{~h}$ & $\beta-(\mathrm{Cm} 242)$ & $3.69 \mathrm{E}+00$ \\
\hline $\mathrm{Cm}-242$ & $5.54 \mathrm{E}+16$ & $162.8 \mathrm{~d}$ & $\beta-(\mathrm{Pu} 238)$ & $4.52 \mathrm{E}+02$ \\
\hline Am-244 & $3.07 \mathrm{E}+16$ & $10.1 \mathrm{~h}$ & $\beta-(\mathrm{Cm} 244)$ & $6.52 \mathrm{E}-01$ \\
\hline $\mathrm{Kr}-85$ & $1.83 \mathrm{E}+16$ & $10.739 \mathrm{y}$ & $\beta-(\mathrm{Rb} 85)$ & $1.26 \mathrm{E}+03$ \\
\hline $\mathrm{Sb}-125$ & $1.26 \mathrm{E}+16$ & $2.75856 \mathrm{y}$ & $\beta-(\mathrm{Te} 125)$ & $3.26 \mathrm{E}+02$ \\
\hline Eu-154 & $9.89 \mathrm{E}+15$ & $8.601 \mathrm{y}$ & $\beta-(\operatorname{Gd} 154)$ & $9.88 \mathrm{E}+02$ \\
\hline Eu-155 & $5.49 \mathrm{E}+15$ & $4.753 \mathrm{y}$ & $\beta-(\operatorname{Gd} 155)$ & $3.01 \mathrm{E}+02$ \\
\hline $\mathrm{Pu}-238$ & $3.36 \mathrm{E}+15$ & 87.7 y & $\alpha-(\mathrm{U} 234)$ & $5.31 \mathrm{E}+03$ \\
\hline $\mathrm{Pu}-240$ & $1.00 \mathrm{E}+15$ & $6561 \mathrm{y}$ & $\alpha-(\mathrm{U} 236)$ & $1.19 \mathrm{E}+05$ \\
\hline $\mathrm{Cm}-244$ & $9.25 \mathrm{E}+14$ & $18.11 \mathrm{y}$ & $\alpha-(\mathrm{Pu} 240)$ & $3.09 \mathrm{E}+02$ \\
\hline Pu-239 & $9.11 \mathrm{E}+14$ & $24110 y$ & $\alpha-(\mathrm{U} 235)$ & $3.97 \mathrm{E}+05$ \\
\hline Sm-151 & $6.70 \mathrm{E}+14$ & $90 \mathrm{y}$ & $\beta-(\operatorname{Eu} 151)$ & $6.88 \mathrm{E}+02$ \\
\hline Am-241 & $3.35 E+14$ & $432.6 \mathrm{y}$ & $\alpha-(\mathrm{Np} 237)$ & $2.64 \mathrm{E}+03$ \\
\hline Tc-99 & $2.45 \mathrm{E}+13$ & $2.111 E+5 y$ & $\beta$-(Ru99) & $3.86 \mathrm{E}+04$ \\
\hline
\end{tabular}

\begin{tabular}{|c|c|c|c|c|}
\hline Am-243 & $1.31 \mathrm{E}+13$ & 7364 y & $\alpha-(\mathrm{Np} 239)$ & $1.78 \mathrm{E}+03$ \\
\hline $\mathrm{Cm}-243$ & $1.30 \mathrm{E}+13$ & $29.1 \mathrm{y}$ & $\alpha-(\mathrm{Pu} 239)$ & $6.83 \mathrm{E}+00$ \\
\hline Am-242 & $6.52 \mathrm{E}+12$ & $16.02 \mathrm{~h}$ & $\beta-(\mathrm{Cm} 242)$ & $1.68 \mathrm{E}+01$ \\
\hline Eu-152 & $5.13 \mathrm{E}+12$ & $13.517 \mathrm{y}$ & $\mathrm{EC}, \beta+(\mathrm{Sm} 152)$ & $7.85 \mathrm{E}-01$ \\
\hline $\mathrm{Zr}-93$ & $3.52 \mathrm{E}+12$ & $1.61 \mathrm{E}+6 \mathrm{y}$ & $\beta-(\mathrm{Nb} 93)$ & $3.78 \mathrm{E}+04$ \\
\hline $\mathrm{Pu}-242$ & $2.14 \mathrm{E}+12$ & $3.75 \mathrm{E}+5 \mathrm{y}$ & $\alpha-(\mathrm{U} 238)$ & $1.46 \mathrm{E}+04$ \\
\hline $\mathrm{Pu}-237$ & $1.26 \mathrm{E}+12$ & $45.64 \mathrm{~d}$ & $\mathrm{EC}(\mathrm{Np} 237)$ & $2.79 \mathrm{E}-03$ \\
\hline Cs-135 & $1.22 \mathrm{E}+12$ & $2.3 \mathrm{E}+6 \mathrm{y}$ & $\beta-(\mathrm{Ba} 135)$ & $2.86 \mathrm{E}+04$ \\
\hline Th-234 & $1.16 \mathrm{E}+12$ & $24.10 \mathrm{~d}$ & $\beta-(\mathrm{Pa} 234)$ & $1.36 \mathrm{E}-03$ \\
\hline $\mathrm{Se}-79$ & $1.16 \mathrm{E}+12$ & $3.27 \mathrm{E}+5 \mathrm{y}$ & $\beta-(\mathrm{Br} 79)$ & $2.28 \mathrm{E}+02$ \\
\hline $\mathrm{Sn}-126$ & $9.06 \mathrm{E}+11$ & $2.3 \mathrm{E}+5 \mathrm{y}$ & $\beta-(\mathrm{Sb} 126)$ & $8.62 \mathrm{E}+02$ \\
\hline U-238 & $8.11 \mathrm{E}+11$ & $4.468 \mathrm{E}+9 \mathrm{y}$ & $\alpha-(\operatorname{Th} 234)$ & $6.52 \mathrm{E}+07$ \\
\hline $\mathrm{U}-236$ & $6.43 \mathrm{E}+11$ & $2.342 \mathrm{E}+7 \mathrm{y}$ & $\alpha-(\operatorname{Th} 232)$ & $2.69 \mathrm{E}+05$ \\
\hline $\mathrm{Pa}-233$ & $5.47 \mathrm{E}+11$ & $26.975 \mathrm{~d}$ & $\beta-(\mathrm{U} 233)$ & 7.12E-04 \\
\hline Np-237 & $5.17 \mathrm{E}+11$ & $2.144 \mathrm{E}+6 \mathrm{y}$ & $\alpha-(\operatorname{Pa} 233)$ & $1.98 \mathrm{E}+04$ \\
\hline Am-240 & $4.52 \mathrm{E}+11$ & $50.8 \mathrm{~h}$ & EC-(Pu240) & 4.77E-05 \\
\hline $\mathrm{Pu}-245$ & $2.02 \mathrm{E}+11$ & $10.5 \mathrm{~h}$ & $\beta-(\mathrm{Am} 245)$ & 4.48E-06 \\
\hline Pd-107 & $1.25 \mathrm{E}+11$ & $6.5 \mathrm{E}+6 \mathrm{y}$ & $\beta-(\operatorname{Ag} 107)$ & $6.58 \mathrm{E}+03$ \\
\hline $\mathrm{Pu}-236$ & $1.18 \mathrm{E}+11$ & $2.858 \mathrm{y}$ & $\alpha-(\mathrm{U} 232)$ & $6.10 \mathrm{E}-03$ \\
\hline Th-231 & $9.82 \mathrm{E}+10$ & $25.52 \mathrm{~h}$ & $\beta-(\mathrm{Pa} 231)$ & $5.00 \mathrm{E}-06$ \\
\hline $\mathrm{Pa}-232$ & $8.97 \mathrm{E}+10$ & $1.32 \mathrm{~d}$ & $\beta-(\mathrm{U} 232)$ & 5.64E-06 \\
\hline $\mathrm{Cm}-245$ & $8.63 E+10$ & $8423 \mathrm{y}$ & $\alpha-(\mathrm{Pu} 241)$ & $1.36 \mathrm{E}+01$ \\
\hline $\mathrm{U}-235$ & $8.31 \mathrm{E}+10$ & $7.04 \mathrm{E}+8 \mathrm{y}$ & $\alpha-(\operatorname{Th} 231)$ & $1.04 \mathrm{E}+06$ \\
\hline Ba-133 & $5.62 \mathrm{E}+10$ & $10.551 \mathrm{y}$ & EC-(Cs133) & $5.94 \mathrm{E}-03$ \\
\hline Th-233 & $5.39 \mathrm{E}+10$ & $21.83 \mathrm{~min}$ & $\beta-(\mathrm{Pa} 233)$ & 4.03E-08 \\
\hline $\mathrm{I}-129$ & $4.81 \mathrm{E}+10$ & $1.57 \mathrm{E}+7 \mathrm{y}$ & $\beta-(\mathrm{Xe129})$ & $7.36 \mathrm{E}+03$ \\
\hline U-234 & $3.01 \mathrm{E}+10$ & $2.455 \mathrm{E}+5 \mathrm{y}$ & $\alpha-(\operatorname{Th} 230)$ & $1.31 \mathrm{E}+02$ \\
\hline $\mathrm{Cm}-241$ & $1.01 \mathrm{E}+10$ & $32.8 \mathrm{~d}$ & EC-(Am241) & $1.65 \mathrm{E}-05$ \\
\hline
\end{tabular}

$* \min =$ minute, $\mathbf{h}=$ hour $, \mathbf{d}=\mathbf{d a y}, \mathbf{y}=$ year 
Table (8): Inventory and activities of actinides and fission products for the core after 50 years from reactor scram

\begin{tabular}{|c|c|c|c|c|}
\hline Radionuclide & $\begin{array}{l}\text { Activity } \\
\text { (Bq/core) }\end{array}$ & $\begin{array}{c}\text { Half-life } \\
\text { (T1/2) }\end{array}$ & Decay Mode & $\begin{array}{c}\text { Inventory } \\
\text { (g/core) }\end{array}$ \\
\hline Cs-137 & $6.23 E+16$ & $30.08 y^{*}$ & $\beta-(\mathrm{Ba} 137)$ & $1.94 \mathrm{E}+04$ \\
\hline Sr-90 & $4.56 \mathrm{E}+16$ & $28.79 \mathrm{y}$ & $\beta-(Y 90)$ & $8.73 E+03$ \\
\hline $\mathrm{Pu}-241$ & $2.22 \mathrm{E}+16$ & $14.329 \mathrm{y}$ & $\beta-(\mathrm{Am} 241)$ & $5.80 \mathrm{E}+03$ \\
\hline Am-241 & $7.40 \mathrm{E}+15$ & $432.6 \mathrm{y}$ & $\alpha(\mathrm{Np} 237)$ & $5.84 \mathrm{E}+04$ \\
\hline $\mathrm{Pu}-238$ & $2.47 \mathrm{E}+15$ & 87.7 y & $\alpha(\mathrm{U} 234)$ & $3.90 \mathrm{E}+03$ \\
\hline $\mathrm{Pu}-240$ & $1.00 \mathrm{E}+15$ & $6561 \mathrm{y}$ & $\alpha(\mathrm{U} 236)$ & $1.19 \mathrm{E}+05$ \\
\hline $\mathrm{Pu}-239$ & $9.17 \mathrm{E}+14$ & $2.411 \mathrm{E}+4 \mathrm{y}$ & $\alpha(\mathrm{U} 235)$ & $4.00 \mathrm{E}+05$ \\
\hline $\mathrm{Kr}-85$ & $7.23 \mathrm{E}+14$ & $10.739 y$ & $\beta-(\mathrm{Rb} 85)$ & $4.97 \mathrm{E}+01$ \\
\hline Sm-151 & $4.62 \mathrm{E}+14$ & $90 \mathrm{y}$ & $\beta-(E u 151)$ & $4.75 E+02$ \\
\hline Eu-154 & $1.76 \mathrm{E}+14$ & $8.601 \mathrm{y}$ & $\beta-(\mathrm{Gd} 154)$ & $1.76 \mathrm{E}+01$ \\
\hline $\mathrm{Cm}-244$ & $1.37 \mathrm{E}+14$ & $18.11 \mathrm{y}$ & $\alpha(\mathrm{Pu} 240)$ & $4.57 \mathrm{E}+01$ \\
\hline Тc-99 & $2.46 \mathrm{E}+13$ & $2.111 \mathrm{E}+5 \mathrm{y}$ & $\beta-(\mathrm{Ru} 99)$ & $3.88 \mathrm{E}+04$ \\
\hline Np-239 & $1.31 \mathrm{E}+13$ & $2.356 \mathrm{~d}^{*}$ & $\beta-(\mathrm{Pu} 239)$ & $1.52 \mathrm{E}-03$ \\
\hline Am-243 & $1.31 \mathrm{E}+13$ & 7364 y & $\alpha(\mathrm{Pu} 240)$ & $1.77 \mathrm{E}+03$ \\
\hline Am-242 & $5.10 \mathrm{E}+12$ & $16.02 \mathrm{~h}^{*}$ & $\beta-(\mathrm{Cm} 242)$ & $1.32 \mathrm{E}+01$ \\
\hline $\mathrm{Cm}-242$ & $4.21 \mathrm{E}+12$ & $162.8 \mathrm{~d}$ & $\alpha-(\mathrm{Pu} 238)$ & $3.44 \mathrm{E}-02$ \\
\hline $\mathrm{Cm}-243$ & $3.87 \mathrm{E}+12$ & $29.1 \mathrm{y}$ & $\alpha-(\mathrm{Pu} 239)$ & $2.03 \mathrm{E}+00$ \\
\hline Eu-155 & $3.36 \mathrm{E}+12$ & $4.753 \mathrm{y}$ & $\beta-(\mathrm{Gd} 155)$ & $1.84 \mathrm{E}-01$ \\
\hline $\mathrm{Pu}-242$ & $2.14 \mathrm{E}+12$ & $3.75 E+5 y$ & $\alpha-(\mathrm{U} 238)$ & $1.46 \mathrm{E}+04$ \\
\hline Cs-135 & $1.22 \mathrm{E}+12$ & $2.3 \mathrm{E}+6 \mathrm{y}$ & $\beta-(\mathrm{Ba} 135)$ & $2.86 \mathrm{E}+04$ \\
\hline Th-234 & $8.11 \mathrm{E}+11$ & $24.1 \mathrm{~d}$ & $\beta-(\mathrm{Pa} 234)$ & $9.47 \mathrm{E}-04$ \\
\hline U-238 & $8.11 \mathrm{E}+11$ & $4.468 \mathrm{E}+9 \mathrm{y}$ & $\alpha-(\operatorname{Th} 234)$ & $6.52 \mathrm{E}+07$ \\
\hline U-236 & $6.45 \mathrm{E}+11$ & $2.342 \mathrm{E}+7 \mathrm{y}$ & $\alpha-(\operatorname{Th} 232)$ & $2.69 \mathrm{E}+05$ \\
\hline Np-237 & $6.11 \mathrm{E}+11$ & $2.144 \mathrm{E}+6 \mathrm{y}$ & $\alpha-(\operatorname{Pa} 233)$ & $2.34 \mathrm{E}+04$ \\
\hline
\end{tabular}

\begin{tabular}{|c|c|c|c|c|}
\hline $\mathrm{Pa}-233$ & $6.11 \mathrm{E}+11$ & $26.975 \mathrm{~d}$ & $\beta-(\mathrm{U} 233)$ & 7.95E-04 \\
\hline U-237 & $5.31 \mathrm{E}+11$ & $6.752 \mathrm{~d}$ & $\beta-(\mathrm{Np} 237)$ & $1.76 \mathrm{E}-04$ \\
\hline Pm-147 & $5.23 \mathrm{E}+11$ & $2.6234 \mathrm{y}$ & $\beta-(\operatorname{Sm} 147)$ & $1.52 \mathrm{E}-02$ \\
\hline U-234 & $4.57 \mathrm{E}+11$ & $2.455 \mathrm{E}+5 \mathrm{y}$ & $\alpha-(\operatorname{Th} 230)$ & $1.99 \mathrm{E}+03$ \\
\hline Eu-152 & $3.81 \mathrm{E}+11$ & $13.517 \mathrm{y}$ & $\mathrm{EC}, \beta+(\mathrm{Sm} 152)$ & $5.84 \mathrm{E}-02$ \\
\hline Pd-107 & $1.25 \mathrm{E}+11$ & $6.5 \mathrm{E}+6 \mathrm{y}$ & $\beta-(\operatorname{Ag} 107)$ & $6.58 \mathrm{E}+03$ \\
\hline $\mathrm{Cm}-245$ & $8.60 \mathrm{E}+10$ & $8423 \mathrm{y}$ & $\alpha(\mathrm{Pu} 241)$ & $1.35 \mathrm{E}+01$ \\
\hline Th-231 & $8.32 \mathrm{E}+10$ & $25.52 \mathrm{~h}$ & $\beta-(\mathrm{Pa} 231)$ & $4.23 \mathrm{E}-06$ \\
\hline U-235 & $8.32 \mathrm{E}+10$ & $7.04 \mathrm{E}+8 \mathrm{y}$ & $\alpha($ Th231) & $1.04 \mathrm{E}+06$ \\
\hline $\mathrm{I}-129$ & $4.85 \mathrm{E}+10$ & $1.57 \mathrm{E}+7 \mathrm{y}$ & $\beta-(\mathrm{Xe129})$ & $7.42 \mathrm{E}+03$ \\
\hline Sb-125 & $3.94 \mathrm{E}+10$ & $2.75856 \mathrm{y}$ & $\beta-(\mathrm{Te} 125)$ & $1.02 \mathrm{E}-03$ \\
\hline Np-238 & $2.30 \mathrm{E}+10$ & $2.099 \mathrm{~d}$ & $\beta-(\mathrm{Pu} 238)$ & $2.39 \mathrm{E}-06$ \\
\hline Cs-134 & $1.07 \mathrm{E}+10$ & $2.0652 \mathrm{y}$ & $\beta-(\mathrm{Ba134})$ & $2.24 \mathrm{E}-04$ \\
\hline $\mathrm{Cm}-246$ & 7.01E+09 & 4706 y & $\alpha(\mathrm{Pu} 242)$ & $6.17 \mathrm{E}-01$ \\
\hline Np-236 & $2.84 \mathrm{E}+07$ & $1.53 \mathrm{E}+5 \mathrm{y}$ & EC(U236) & $5.82 \mathrm{E}-02$ \\
\hline Xe-135 & $1.93 \mathrm{E}+07$ & $9.14 \mathrm{~h}$ & $\beta-(\mathrm{Cs} 135)$ & $2.05 \mathrm{E}-10$ \\
\hline I-135 & $1.69 \mathrm{E}+07$ & $6.58 \mathrm{~h}$ & $\beta-(\mathrm{Xe135})$ & $1.29 \mathrm{E}-10$ \\
\hline $\mathrm{Xe}-133$ & $1.55 \mathrm{E}+07$ & $5.2475 \mathrm{~d}$ & $\beta-(\mathrm{Cs} 133)$ & $2.24 \mathrm{E}-09$ \\
\hline $\mathrm{Pu}-236$ & $3.29 \mathrm{E}+06$ & $2.858 \mathrm{y}$ & $\alpha(\mathrm{U} 232)$ & $1.70 \mathrm{E}-07$ \\
\hline Cf-249 & $1.34 \mathrm{E}+05$ & $351 \mathrm{y}$ & $\alpha(\mathrm{Cm} 245)$ & $8.85 \mathrm{E}-07$ \\
\hline $\mathrm{Pu}-244$ & $1.09 \mathrm{E}+05$ & $8.13 \mathrm{E}+7 \mathrm{y}$ & $\alpha(\mathrm{U} 240)$ & $1.61 \mathrm{E}-01$ \\
\hline $\mathrm{Cm}-248$ & $2.35 \mathrm{E}+04$ & $3.48 \mathrm{E}+5 \mathrm{y}$ & $\alpha(\mathrm{Pu} 244)$ & $1.50 \mathrm{E}-04$ \\
\hline $\mathrm{Pu}-243$ & $1.48 \mathrm{E}+04$ & $4.956 \mathrm{~h}$ & $\beta-(\mathrm{Am} 243)$ & $1.54 \mathrm{E}-13$ \\
\hline $\mathrm{Cm}-247$ & $1.48 \mathrm{E}+04$ & $1.56 \mathrm{E}+7 \mathrm{y}$ & $\alpha(\mathrm{Pu} 243)$ & 4.43E-03 \\
\hline
\end{tabular}

$* \mathbf{h}=$ hour, $\mathbf{d}=$ day, $\mathbf{y}=$ year 


\section{CONCLUSIONS}

The nuclear spent fuel was characterized by the estimation of the inventories and activities of the radionuclides using MCNPX 2.7 code for FD-U1 accident. The MCNPX results were validated with the experimental measurements which were carried out by JNES. Also, the activities of the radionuclides were compared with JAEA calculations using ORIGEN2 code. The activities of the source term composition were categorized according to the NUREG-1465.These information are important for the assessment of the radiological releases to the environment.

As the initial amount of uranium fuel in the core in the last reloading before the accident was about 69 ton, and the calculated amount of the uranium fuel at the time of the accident was about 66.51 ton, then, the rest of the uranium fuel mass was depleted for about 165 operating days according to JAEA. Also, the fission products mass was found to be about 2.49 ton. In addition, the total estimated mass of the core composition was about 121.48 ton, which includes the depleted fuel, control rods and structural materials.

The results showed that, at the time of accident, the total activity and the specific activity of the actinide and the non-actinide in the core was $9.86 \mathrm{E}+19 \mathrm{~Bq}$ and $1.84 \mathrm{E}+15 \mathrm{~Bq} / \mathrm{g}$, respectively; and after 50 years cooling time, the total activity and the specific activity of the actinide and the non-actinide in the core was $1.89 \mathrm{E}+17 \mathrm{~Bq}$ and $5.86 \mathrm{E}+12 \mathrm{~Bq} / \mathrm{g}$, respectively. We can notice the slow activity reduction of the long-lived radionuclides after 50 years from the accident.

The information on the inventories and activities for the actinides and fission products can support the recovery program for Fukushima Daiichi - Unit -1.

\section{ACKNOWLEDGEMENTS}

The authors acknowledge and thank the IAEA for the sponsorship of this research work under a Coordinated Research Project Code: CRP-T13015, entitled, Management of Severely Damaged Spent Fuel and Corium Research Project, and the Egyptian Atomic Energy Authority (EAEA) for supporting this work and using the licensed MCNPX code.

\section{REFERENCES}

[1] Mark Holt, et al., Fukushima Nuclear Disaster, January 18, (2012).
[2] Fukushima, one year later, initial analyses of the accident and its consequences, Report IRSN/DG/2012-003, March 12, (2012).

[3] Tokyo Electric Power Company (TEPCO), November 30, (2011), The Evaluation Status of Reactor Core Damage at Fukushima Daiichi Nuclear Power Station Units1-3,pp.621,http://www.tepco.co.jp/en/nu/fukushimap/images/handouts_111130_04-e.pdf.

[4] Earthquake Report No.91, Japan Atomic Industrial Forum (JAIF), 25 May, (2011).

[5] Yoshiaki Tsukuda, et al., Proving Test on Thermal-Hydraulic Performance of BWR Fuel Assemblies, Journal of Nuclear Science and Technology, Atomic Energy Society of Japan, (2002), Vol. 1, Issue 4.

[6] K. Suyama, et al., Burn-up Credit Criticality Safety Benchmark Phase III-C Nuclide Composition and Neutron Multiplication Factor of a Boiling Water Reactor Spent Fuel Assembly for Burn-up Credit and Criticality Control of Damaged Nuclear Fuel, Nuclear Science Committee, OECD Nuclear Energy Agency, March, (2016).

[7] Katsuhiro TSUDA, Akira ITAMI, Yuichiro KUBO et al., Analysis of Core Stability Measurement Data of Advanced 9 $\times 9$ Fuel Assembly in a BWR Core, Journal of Nuclear Science and Technology, 15 Mar., (2012).

[8] Yoshitaka NAITO and Hiroshi OKUNO, OECD/NEA- Burrup Credit Criticality Benchmark- Phase IIIB, Nuclide Composition and Neutron Multiplication Factor of BWR Spent Fuel Assembly Japan Atomic Energy Research Institute, November, (1996).

[9] Toru YAMAMOTO and Yuichiro KANAYAMA, Lattice Physics Analysis of Burn-ups and Isotope Inventories of $\mathrm{U}, \mathrm{Pu}$, and $\mathrm{Nd}$ of Irradiated BWR9 $\times 9-9 \mathrm{UO}_{2}$ Fuel Assemblies, Journal of Nuclear Science and Technology, (2012), Jan.

[10] C. Markley, E.L. Tipton, J. Winterle et al., " $\beta$ Soar: A flexible tool for analyzing disposal of nuclear waste," Proceedings of the 2011 International High-Level Radioactive Waste Management Conference (IHLRWM), Albuquerque, New Mexico, April 10-14, (2011). 
[11] IAEA, Derivation of the Source Term and Analysis of the Radiological Consequences of Research Reactor Accident, (2008), safety report series No.53, IAEA, Vienna.

[12] Ralston W. Barnard, Review of Radionuclide Source Terms used for Performance Assessment Analyses, ASND 92-2431, June (1993).

[13] Jon M.Schwantes, Christopher R.Orton , Richard A.Clark, Analysis of a Nuclear Accident: Fission and Activation Product Releases from the Fukushima Daiichi Nuclear Facility as Remote Indicators of Source Identification, Extent of Release and State of Damaged Spent Nuclear Fuel, (2012), J. Environ.Sci.Technol.46,16, pp.8621-8627.

[14] Vidya Thorat, et al., Characterization of High Level Nuclear Waste Solutions from Different Origin, (2019), J. Radio-analytical and Nuclear Chemistry 320(3), May .

[15] J.C.de.La Rosa Blue, P.Mc Minn, A. Grah, Analysis of the Inherent Response of Nuclear Spent Fuel Pools, (2019), Annals of Nuclear Energy, Vol.124, pp.295-326.

[16] MCNPX User's Manual Version 2.7.0, April, (2011).

[17] Toru YAMAMOTO, Compilation of Measurement and Analysis Results of Isotopic Inventories of Spent BWR Fuels, Japan Nuclear Energy Safety Organization, (2009).

[18] Benchmark Study of the Accident at the Fukushima Daiichi Nuclear Power Plant (BSAF Project), Phase I Summary Report, March (2015).

[19] Compendium of Material Composition Data for Radiation Transport Modeling, PNNL-15870 Rev. 1, March 4, (2011).

[20] https://www.oecd-nea.org/science/wpncs/ Publications/ref-val-criticality-safety/
[21] T.Yamamoto, Y. Kanayama, Lattice Physics Analysis of Burnups and Isotope Inventories of $\mathrm{U}$, $\mathrm{Pu}$, and $\mathrm{Nd}$ of Irradiated BWR $9 \times 9-9 \mathrm{UO}_{2}$ Fuel Assemblies, (2008), J. Nucl. Sci. and Technol., 45 (6), 547.

[22] T. Yamamoto, Y. Kanayama, Nuclear Analysis of PIE Data of Irradiated BWR $8 \times 8-2$ and $8 \times 8-4$, $\mathrm{UO}_{2}$ Fuel Assemblies, (2008), J. Nucl. Sci.Tech.

[23] IAEA, the Fukushima Daiichi Accident Technical, Safety Assessment, August, (2015),Vol.2, Vienna.

[24] Kenji NISHIHARA, Hiroki IWAMOTO and Kenya SUYAMA, Estimation of Fuel Compositions in Fukushima-Daiichi Nuclear Power Plant, Division of Nuclear Data and Reactor Engineering, Japan Atomic Energy Agency, (2012).

[25] IAEA, the Fukushima Daiichi Accident Technical Volume 4, Radiological Consequences, (2015), Vol.4, Vienna.

[26] IAEA, the Fukushima Daiichi Accident Technical Volume 1 Description and Context of the Accident, (2015), Vol.1,Vienna.

[27] Shuhei Miwaa et al., Research Program for the Evaluation of Fission Product and Actinide Release Behavior, Focusing on Their Chemical Forms, The Fourth International Symposium on Innovative Nuclear Energy Systems, INES-4, Energy Proceedings, (2015), 71 (68-181).

[28] Modeling and validation of the Fuel Depletion and Burn-up of the OSU research reactor using MCNPX/CINDER'90.

[29] Fumiya Tanabe, Analysis of Core Melt Accident in Fukushima Daiichi-Unit 1 Nuclear Reactor, (2011), J. Nucl. Sci. and Tech. Vol. 48, No. 8, p. 1135-1139. 